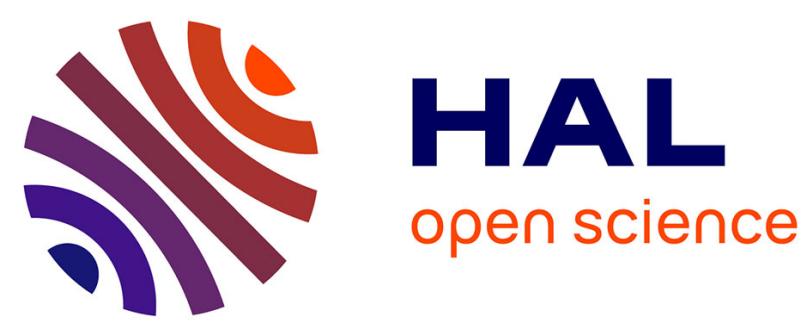

\title{
Le traitement des connecteurs dans les instructions officielles et les manuels (français L1 / anglais L2
}

Marie-Laure Elalouf, Anne Trevise

\section{To cite this version:}

Marie-Laure Elalouf, Anne Trevise. Le traitement des connecteurs dans les instructions officielles et les manuels (français L1 / anglais L2. Revue Française de Linguistique Appliquée, 2011, Les connecteurs: description, traduction, apprentissage, 16 (2), pp.121-140. halshs-00668554

\section{HAL Id: halshs-00668554 \\ https://shs.hal.science/halshs-00668554}

Submitted on 9 Feb 2012

HAL is a multi-disciplinary open access archive for the deposit and dissemination of scientific research documents, whether they are published or not. The documents may come from teaching and research institutions in France or abroad, or from public or private research centers.
L'archive ouverte pluridisciplinaire HAL, est destinée au dépôt et à la diffusion de documents scientifiques de niveau recherche, publiés ou non, émanant des établissements d'enseignement et de recherche français ou étrangers, des laboratoires publics ou privés. 


\section{Le traitement des connecteurs dans les Instructions officielles et les manuels}

(français L1, anglais L2)

Marie-Laure Elalouf (Université de Cergy-Pontoise, EMA)

et Anne Trévise (Université de Paris Ouest Nanterre la Défense, UMR 7114 Modyco)

Le terme de «connecteur» fait partie de ces termes relativement rares, qui se sont implantés dans la terminologie scolaire et dans l'usage des professeurs sans susciter de polémique, à la différence d'un terme comme «déictique» par exemple. Pourtant, son intérêt didactique n'est pas établi. Il est en effet utilisé, dans les programmes de français $\left(\mathrm{L}^{1}{ }^{1}\right)$ et d'anglais (L2) comme un terme englobant, appliqué à des unités ayant des fonctionnements linguistiques très différents, réunies sur la base de rapprochements sémantiques approximatifs. Nous montrerons que le terme «connecteur», tel qu'il est utilisé, dans les textes officiels ou les manuels, ne permet de cerner ni la spécificité ni la fonctionnalité des usages dans les deux langues.

\section{Le français $\mathrm{L1}$}

Nous décrirons comment le terme est entré dans les programmes grâce à un aperçu historique. Puis nous éclairerons les référents théoriques implicites à partir desquels s'est élaborée la définition scolaire des connecteurs et ceux qui ont été écartés. Enfin, nous montrerons, à travers l'analyse d'un chapitre de manuel, les limites de cet usage terminologique.

\subsection{Le traitement des connecteurs dans les nomenclatures et programmes}

Si l'on considère l'histoire des terminologies grammaticales et des programmes scolaires, on peut distinguer trois périodes d'inégales durées, avec un décalage d'une dizaine d'années entre le secondaire et le primaire.

\subsubsection{Première période : les conjonctions}

La première période, la plus longue, va de la nomenclature de 1910, prise comme origine, aux nomenclatures de 1975 pour le secondaire et 1985 pour le primaire. Les parties du discours y sont réparties en mots variables et mots invariables. Parmi ceux-ci, on trouve les

\footnotetext{
1 terme nettement préférable à celui de « langue maternelle ».
} 
conjonctions et locutions conjonctives, dont la mémorisation est requise sous forme de liste pour l'analyse des propositions. L'évolution sémantique du terme «coordination » est caractéristique d'une démarche analytique dans laquelle la conjonction est identifiée et dotée d'une fonction syntaxique et sémantique à la charnière de deux segments. On a d'abord employé le terme « coordination » pour désigner l'identité de rang, qu'elle soit ou non marquée par un morphème. C'est ce qu'enregistre la nomenclature de 1910 :

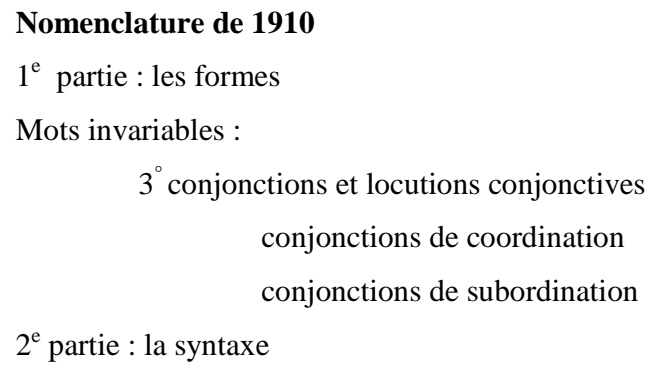

Division des propositions

$3^{\circ}$ Propositions subordonnées.

NB. Les propositions principales et subordonnées peuvent être coordonnées.

Puis l'on a réservé le terme « coordination » à la présence d'une conjonction prise dans une liste rituelle de sept mots, par opposition à la juxtaposition : dans la nomenclature de 1975 pour le secondaire, le Nota bene de 1910 devient : «Les phrases comme les propositions, peuvent être juxtaposées ou coordonnées ». Les programmes de 1985 pour le primaire font de même.

Dans cette première période, l'identification d'une classe de mots invariables appelés conjonctions est au service de l'analyse des propositions. Les exercices les plus courants consistent à repérer, sur des phrases isolées, les verbes conjugués et les conjonctions puis à délimiter les propositions et à les nommer.

\subsubsection{Deuxième période : les mots de liaison}

La seconde période commence explicitement avec les programmes de 1985 pour le secondaire et ceux de 1995 pour le primaire, mais elle est annoncée par une remarque dans les programmes de 1977 pour le collège :

Programmes du collège unique, 1977

$6^{\mathrm{e}}-5^{\mathrm{e}}$

Enrichissement du vocabulaire : mots grammaticaux

L'étude portera sur les mots grammaticaux indispensables à la pratique et à l'intelligence de la langue.

$4^{\mathrm{e}}-3^{\mathrm{e}}$ 
Enrichissement du vocabulaire : mots grammaticaux

On insistera sur le rôle (...) des conjonctions et locutions conjonctives, adverbes et locutions adverbiales comme instruments de rapports temporels et logiques.

Cette remarque rompt avec la démarche analytique précédente et adopte, comme c'est précisé pour la $6^{\mathrm{e}}$ et la $5^{\mathrm{e}}$, la perspective de «la pratique et de l'intelligence de la langue » : il s'agit de faire acquérir aux élèves des termes qu'ils maîtrisent approximativement en les faisant réfléchir à leurs emplois.

Les programmes de la seconde période reprennent à Aristote le terme de « mot de liaison » - sundesmoï - pour décrire les faits d'enchaînement au niveau du texte : les mots ainsi réunis le sont sur la base d'une fonction commune, non plus syntaxique mais textuelle, et relevant soit des conjonctions (sans préciser lesquelles) soit des adverbes :

\section{Programmes du collège, 1985}

$6^{e}-5^{e}$

La phrase

La phrase simple et la phrase complexe

La phrase simple et la phrase complexe

Juxtaposition, coordination, subordination. Propositions indépendantes, principales, subordonnées.

Les conjonctions de coordination. Les conjonctions de subordination et le pronom relatif.

La phrase et le texte

La liaison des phrases. Mots de liaison, mots de reprise (anaphoriques).

\section{$4^{e}-3^{e}$}

La phrase complexe

La phrase et le texte

L'enchaînement des phrases et la cohérence du texte : mots de liaison (adverbes, conjonctions), mots de reprise (pronoms, substituts lexicaux), éléments reprise et éléments nouveaux d'une phrase à l'autre (thème et développement).

Le texte

Les types de textes : narratif, descriptif, documentaire, argumentatif, etc.

Deux autres caractéristiques du texte sont introduites en même temps: les procédés anaphoriques («mots de reprise») et la progression thématique ("éléments nouveaux d'une phrase à l'autre »). Les programmes de 1995 pour le primaire adoptent clairement une démarche du tout à la partie, dans laquelle l'organisation du texte (et notamment le rôle de la ponctuation) prime sur l'identification des conjonctions: la périphrase «mots permettant l'agencement des parties entre elles » est une glose de l'expression «mots de liaison » du collège.

\section{Programmes de l'école primaire, 1995}




\section{Cycle 2}

Le texte, le paragraphe, la phrase dans le texte, la ponctuation

\section{Cycle 3}

L'organisation et la cohérence du texte

Rôle du chapitre, du paragraphe, des mots permettant l'agencement des parties entre elles.

La phrase

Les phrases simples et construites par juxtaposition, coordination ou subordination.

Les classes de mots

Les prépositions, les adverbes (Les conjonctions ne sont pas citées.)

L'introduction de l'expression «mots de liaison»s'accompagne d'une redéfinition des finalités de l'étude de la langue : améliorer les compétences de réception et de production des textes.

\subsubsection{Troisième période : les connecteurs}

La troisième période commence avec l'introduction du terme de «connecteur » dans la terminologie de 1997 :

Le texte

Mots de liaison ; connecteurs.

Connecteurs logiques : Car, en effet, c'est pourquoi, donc, parce que, puisque, d'ailleurs, or, mais, pourtant, cependant, certes... mais, aussi, ainsi, nous en déduisons que, d'une part... d'autre part, etc.

Indicateurs temporels : Aujourd'hui, demain, hier, maintenant, alors, ce jour-là, le lendemain, la veille, ensuite, puis, d'abord, auparavant, plus tard, les compléments circonstanciels exprimant le temps, etc.

Indicateurs spatiaux : ici, là, là-bas, devant, derrière, dessus, dessous, au-delà, plus loin, plus haut, plus bas, les compléments circonstanciels exprimant le lieu, etc.

La phrase

Enchâssement, subordination, coordination, parataxe

Classes de mots

Conjonctions de subordination: que, parce que, pour que, lorsque, pourvu que, quoique, comme, quand, si, etc.

Conjonction de coordination: car, donc, et, mais, ni, or, ou. 
Le terme de «connecteur » est donné comme un équivalent de «mot de liaison » mais connaît une triple extension que décrivent sans l'expliquer Maingueneau et Pellet (2005, 139) dans leur guide intitulé Les notions grammaticales au collège et au lycée :

Au plan syntaxique elle [englobe] tous les éléments susceptibles d'établir un lien à l'intérieur de la phrase (entre propositions) aussi bien qu'entre phrases, incluant les conjonctions de subordination. Au plan sémantique, par analogie avec les relations logiques et temporelles (d'abord, ensuite, puis) la grammaire textuelle l'a appliquée à l'organisation spatiale (devant, derrière, à gauche).

Une troisième extension, non signalée par les auteurs, est observée : le terme «connecteur » ne désigne plus seulement des morphèmes ou des séquences grammaticalisés, mais des syntagmes libres, voire des phrases. Ainsi, dans la terminologie de 1997, on trouve parmi les connecteurs dits «logiques» des conjonctions, des adverbes, mais aussi des phrases (nous en déduisons que), réunis sur la base d'une analogie sémantique qui relève de l'argumentation plus que de la logique. De même, les indicateurs spatiaux et temporels regroupent des adverbes et des syntagmes libres: les compléments circonstanciels exprimant le temps ou le lieu. L'extension de la classe sert l'identification grossière des types de textes argumentatif, narratif et descriptif. La distinction connecteurs/ indicateurs n'est pas retenue dans les programmes de 1996-99 pour le collège qui étendent l'emploi du terme aux connecteurs spatiaux-temporels.

Les programmes 2008 pour l'école et 2009-2012 pour le collège introduisent une rupture par rapport aux précédents en préconisant une démarche analytique centrée sur l'identification des classes de mots dans la phrase jusqu'en $4^{\mathrm{e}}$. Ils diffèrent ainsi l'introduction de la grammaire de texte et du terme de «connecteur», mais sans en modifier l'acception élargie :

\section{Programmes de l'école primaire, 2008}

Classes de mots : toutes sauf conjonction

Les propositions

Phrase simple/ complexe, proposition indépendante (coordonnée, juxtaposée) et proposition subordonnée.

\section{Programmes du collège, 2009-2012}

$$
5^{e}
$$

L'analyse de la phrase

La phrase complexe : la coordination et la subordination

Les classes de mots

Conjonctions de coordination

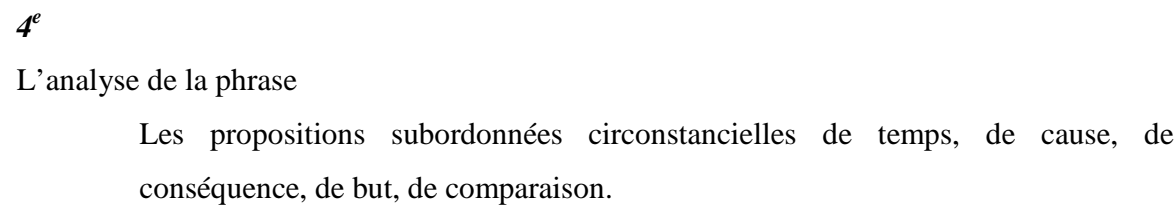
conséquence, de but, de comparaison. 


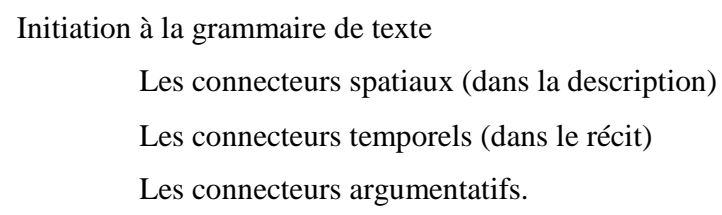

Dans le même temps, en couplant l'étude des connecteurs avec celles des subordonnées circonstancielles, ils renforcent l'assimilation entre leurs valeurs et l'expression des circonstances.

Nous avons ainsi suivi l'itinéraire d'un terme dont l'introduction est liée aux limites d'une description de la conjonction sur le plan uniquement phrastique, comme le proposait la nomenclature de 1975 : établir un rapport logique entre deux unités significatives de même fonction ou dans un rapport de dépendance syntaxique. Mais l'extension de ce terme échappe en partie aux explications linguistiques. Elle est aussi à rechercher du côté des pratiques scolaires. La maîtrise de la langue est devenue l'objectif prioritaire de l'enseignement du français, de l'école primaire au lycée, sans que les contenus de la grammaire de phrase n'aient été modifiés (Combettes 2009) : faire repérer les connecteurs pour identifier les types de textes est apparu facile à enseigner et à évaluer. Mais le modèle de référence pour l'étude de la langue restant la phrase, on a rabattu le nouveau sur l'ancien, c'est-à-dire les connecteurs sur les compléments circonstanciels, le texte étant conçu comme un élargissement de la phrase.

\subsection{Les référents théoriques implicites}

Quels référents théoriques implicites sous-tendent cet itinéraire? Nous nous sommes tournée vers des ouvrages accessibles à des enseignants du secondaire, préconisés dans la préparation des concours internes et externes.

\subsubsection{L'introduction de l'expression « mot de liaison »}

L'expression « mots de liaison » apparaît dans les programmes de 1985 pour le secondaire sans définition, mais la description qui y est proposée correspond à la définition que donne des connecteurs la Grammaire d'aujourd'hui (Arrivé, Gadet \& Galmiche 1984) :

CONNECTEUR: Terme qui permet de regrouper les usages interphrastiques des conjonctions de coordination, et de certains adverbes ou assimilés qui jouent le même rôle en tête de phrase : et, or, puis, cependant, en fin de compte... sont des connecteurs.

\subsubsection{La triple extension du concept de connecteur}


La Grammaire méthodique du français (Riegel, Pellat \& Rioul 1994), plus récente, signale la pluralité des usages du terme, en opposant un sens restreint et un sens élargi. Le sens restreint ne coöncide pas avec la définition de la Grammaire d'aujourd'hui ; il correspond à une définition syntaxique qui lie étroitement présence d'une conjonction et établissement d'une liaison au sein de la phrase complexe. Le sens élargi regroupe des procédés très divers sous une fonction très générale : l'organisation du texte. La définition retenue par les auteurs pour en limiter l'extension est celle de Schneuwly (1989) qui associe deux critères : une position syntaxique périphérique - ce sont des unités linguistiques qui ne font pas partie intégrante des propositions, plutôt en début de propositions - et une fonction textuelle. Ce faisant, cette définition place dans un continuum la phrase et le texte en n'envisageant que les relations entre propositions. L'extension des connecteurs aux conjonctions de subordination est ainsi permise.

\subsubsection{Les référents théoriques transformés ou écartés}

Les deux autres extensions observées dans la Terminologie grammaticale de 1997 peuvent s'expliquer par une lecture réductrice des travaux de pragmatique. Si l'on se réfère à l'article de Christian Plantin dans le Dictionnaire d'analyse du discours (Charaudeau \& Maingueneau 2002), on trouve la description suivante :

\section{Connecteur}

En adoptant un point de vue pragmatique et textuel, on gagne à placer sur un continuum plusieurs types de connecteurs qui remplissent certes une même fonction de liage entre unités de rang différent (propositions ou paquets de propositions) mais qui (1) soit assurent cette simple fonction de connexion, (2) soit ajoutent un rôle de marquage de (re)prise en charge énonciative, (3) soit complètent ces deux fonctions par une orientation argumentative marquée.

1. Segmenter et relier : la simple connexion (les organisateurs)

a. Ceux qui ordonnent les éléments de la représentation discursive : organisateurs spatiaux (à droite, à gauche, devant, derrière, dessus, dessous, plus loin, d'un côté-de l'autre...) ; organisateurs temporels (alors, ensuite, [et] puis, après, la veille, le lendemain, trois jours plus tard, maintenant...)

2. Marquer énonciativement une portion de texte : connexion combinée à une prise en charge énonciative

a. Connecteurs de reformulation (c'est-à-dire, autrement dit, [N1] c'est/ s'appelle un [N2])

b. Organisateurs marqueurs de structuration de la conversation (bon, ben, alors) et autres phatiques (tu sais, tu vois, euh...)

3. Orienter argumentativement : les connecteurs argumentatifs déclenchent un retraitement du contenu propositionnel soit comme un argument soit comme une conclusion, soit comme un argument chargé d'étayer ou de renforcer une inférence, soit comme un contre argument.

a. Argumentatifs et concessifs (mais, pourtant, cependant, certes, toutefois, quand même) 
b. Introducteurs d'explication et de justification (car, parce que, puisque, si, c'est que)

c. Si hypothétique (si-alors)

d. Simples marqueurs d'un argument (même, d'ailleurs, de plus, non seulement).

Ce qui est décrit ici comme un processus interprétatif qui organise et transforme la représentation discursive se traduit dans les programmes et les manuels par une liste de connecteurs temporels, spatiaux ou logiques. Disparaît en même temps l'idée que cette fonction peut s'exercer entre des propositions ou des «paquets de propositions», voire repose sur un acte d'énonciation ou sur l'implicite, comme le rappelle l'analyse de Moeschler et Auchlin $(2009,190)$.

Or, si l'étude de l'implicite figure dans les programmes de collège et de lycée, elle n'est pas mise en relation avec un type de connexion fréquent à l'oral et qui peut être source de dysfonctionnement dans les écrits scolaires voire universitaires (Masseron 2004).

\subsubsection{Questions pendantes pour la didactique}

Quelques questions, rarement abordées dans les ouvrages de référence des enseignants, mériteraient d'être clarifiées. La mise au jour du fonctionnement des connecteurs impliquet-elle une redéfinition de la classe grammaticale des conjonctions ? Cette classe désignant une fonction et non une nature, est-elle superflue, comme le considère Le Goffic (1993) pour qui «les termes conjonctifs ou connecteurs ne cessent pas pour autant d'être adverbes ou pronoms », ou faut-il rendre compte du processus de grammaticalisation des segments fonctionnant comme connecteur en réintroduisant le terme de «particule» (Touratier 2000) ? La prise de conscience de ce processus est rendue difficile car l'interprétation sémantique des éléments périphériques à la construction phrastique n'est jamais associée à des manipulations dans les présentations scolaires. Or, certains connecteurs peuvent être détachés en position thématique, former le cadre de l'énoncé et être encadrés par c'est ... que en position rhématique, comme les compléments circonstanciels, alors que d'autres sont impropres à cet emploi :

(1) Alors, le train a déraillé/ c'est alors que le train a déraillé

(2) Puis le train a déraillé/ *c'est puis que le train a déraillé.

Ce type de manipulation permettrait de faire la distinction entre les adverbes qui jouent un rôle de connexion tout en ayant une valeur circonstancielle et les éléments extraprédicatifs de nature diverse. Autre question rarement abordée, la mise au jour du fonctionnement des connecteurs implique-t-elle une redéfinition des unités d'analyse de l'oral et de l'écrit dans l'usage scolaire? On constate en effet que les connecteurs de l'oral sont singulièrement absents des listes figurant dans les manuels, ce qui va de pair avec une approche de l'oral 
limitée aux niveaux de langue, alors qu'une prise en compte des travaux sur la micro- et la macro-syntaxe fournirait des outils de comparaisons plus sûrs entre l'écrit et l'oral.

\subsection{Une illustration des transpositions didactiques les plus courantes}

Dans un article de 1998, Garcia-Debanc montrait les limites d'une pratique très répandue : «observation d'un texte très chargé en connecteurs variés, puis (...) tableau récapitulatif des divers types de connecteurs possibles » et exercices d'application visant à rétablir des connecteurs supprimés. Force est de constater que cette pratique reste très majoritaire et que, loin de guider les élèves dans l'interprétation et l'utilisation maîtrisée des connecteurs, elle les place devant des contradictions. Dans le manuel de $3^{\text {ème }}$ que nous avons retenu pour sa diffusion, L'atelier du langage (2009), la définition sémantique donnée correspond à la triple extension que nous avons décrite : extension aux conjonctions de subordination, aux indicateurs spatiaux et à tout groupe à valeur de circonstance, ce que met en évidence un premier tableau à double entrée.

\begin{tabular}{|c|c|c|c|}
\hline Classes grammaticales & Connecteurs temporels & $\begin{array}{l}\text { Connecteurs } \\
\text { spatiaux }\end{array}$ & $\begin{array}{l}\text { Connecteurs } \\
\text { logiques }\end{array}$ \\
\hline $\begin{array}{l}\text { Adverbe ou locution } \\
\text { adverbiale }\end{array}$ & $\begin{array}{l}\text { Alors, puis, ensuite, } \\
\text { d'abord, cependant... }\end{array}$ & $\begin{array}{l}\text { Ici, là, derrière, } \\
\text { dessous... }\end{array}$ & $\begin{array}{l}\text { Cependant, pourtant, } \\
\text { par conséquent... }\end{array}$ \\
\hline $\begin{array}{l}\text { Conjonction } \\
\text { coordination }\end{array}$ & $\mathrm{e} t$ & & et, donc, car \\
\hline $\begin{array}{l}\text { Conjonction } \\
\text { subordination }\end{array}$ & $\begin{array}{l}\text { Dès que, quand, } \\
\text { lorsque, alors que, } \\
\text { comme... }\end{array}$ & & $\begin{array}{l}\text { Parce que, si bien } \\
\text { que... }\end{array}$ \\
\hline $\begin{array}{l}\text { Groupe introduit par } \\
\text { une préposition }\end{array}$ & $\begin{array}{l}\text { Après le dîner, avant } \\
\text { de partir, suite à ces } \\
\text { événements... }\end{array}$ & $\begin{array}{l}\text { Autour de moi, au } \\
\text { nord, sur cette table, } \\
\text { derrière le bureau... }\end{array}$ & $\begin{array}{lr}\text { Malgré cette } \\
\text { discussion, grâce à } \\
\text { cela... }\end{array}$ \\
\hline
\end{tabular}

Sur le plan syntaxique, les segments ainsi réunis sont disparates. Par exemple, alors commute avec après le dîner mais non avec alors que. Un second tableau, à entrée sémantique, propose des listes de prépositions (et non de groupes prépositionnels), conjonctions et adverbes. Ce dernier illustre bien l'assimilation des valeurs des connecteurs aux compléments circonstanciels - ainsi l'expression du but est-elle introduite par analogie - et le nivellement des valeurs argumentatives et des procédés de construction du texte - la cause y figure sur le même plan que «l'addition et les étapes du raisonnement ». S'ajoute une mise en garde qui entre en contradiction avec la présentation: «Il ne faut pas confondre les termes connecteurs et compléments circonstanciels. Les connecteurs jouent un rôle dans le texte, alors que les compléments circonstanciels désignent une fonction grammaticale dans la phrase ». 
Les insuffisances d'une telle leçon n'apparaissent guère quand on se tourne vers les exercices : il suffit de relever des connecteurs et de les classer, ce que l'on peut faire en se reportant aux listes données. Elles éclatent en revanche quand on se reporte aux travaux

d'écriture et de lecture. À vos plumes propose deux exercices, non d'écriture mais de reconstitution de texte : la consigne de l'exercice 9 «complétez ces phrases en utilisant le connecteur qui convient », laisse penser qu'un seul connecteur est possible tandis que celle de l'exercice 10, sur un extrait traduit, invite à la variation stylistique sans prise en compte des valeurs différentielles de termes: «complétez le texte suivant avec des connecteurs logiques d'opposition ou de concession en faisant varier les termes (cependant, pourtant, quoique, mais, néanmoins, toutefois...) ». Quant à l'exercice d'observation qui inaugure la leçon, un extrait du Colonel Chabert, il met les élèves devant une authentique complexité sans leur donner les moyens de la débrouiller.

\footnotetext{
Quoique la mémoire de ces moments soit bien ténébreuse, quoique mes souvenirs soient bien confus, malgré les impressions de souffrances encore plus profondes que je devais éprouver et qui ont brouillé mes idées, il y a des nuits où je crois encore entendre ces soupirs étouffés! Mais il y a eu quelque chose de plus horrible que les cris, un silence que je n'ai éprouvé nulle part, le vrai silence du tombeau.
}

Dans le début du texte apparaissent quoique, malgré et mais et les élèves peuvent en retrouver la valeur dans le second tableau : «l'opposition ou la concession». Mais cette équivalence se révèle insuffisante. Les trois groupes antéposés «quoique la mémoire de ces moments soit bien ténébreuse, quoique mes souvenirs soient bien confus, malgré les impressions de souffrances encore plus profondes que je devais éprouver et qui ont brouillé mes idées » sont à interpréter par rapport à «il y a des nuits où je crois encore entendre ces soupirs étouffés! » Ils pourraient être postposés : leur place est contrainte par l'ordre du texte et notamment l'anaphore dite infidèle « ces soupirs étouffés ... les cris ». Le mais qui suit ne se situe pas sur le même plan. Il porte sur l'ensemble de ce qui précède ; la relation d'opposition est à restituer à partir du texte et d'une inférence qu'il conduit à faire rétroactivement, en raison de la présence du comparatif de supériorité : «〈c'était horrible〉 Mais il y a quelque chose de plus horrible encore ». Les autres connecteurs surlignés ne trouvent pas d'explication dans la leçon.

On est porté à penser que des élèves ayant uniquement appris à identifier les conjonctions dans la phrase et les connecteurs dans le texte auront peu réfléchi aux fonctionnements syntaxiques et discursifs guidant l'interprétation et la production de textes dans leur langue 1 et risquent d'être démunis lorsqu'ils aborderont une langue 2.

\section{L'anglais L2}

Dans les textes officiels pour l'enseignement de l'anglais, on retrouve des positions caractérisées par un éclectisme que ne justifient ni une réelle approche didactique des 
processus de l'apprentissage d'une L2, ni un impact escompté de l'enseignement sur ces processus.

En effet, à chaque relecture des textes dits « officiels », programmes et accompagnements, il y a deux postures qui frappent le chercheur en didactique :

- d'une part l'autisme par rapport au filtre préalable omniprésent de la L1, attitude çà-et-là démentie par des peurs avouées de calques, ou par une transdisciplinarité volontariste, misant sur une rétroaction sur la maîtrise de la L1, sans aucune justification psycholinguistique ;

- d'autre part, les formules incantatoires sur les liens non prouvés entre savoirs et savoir-faire : les savoir-faire par l'imprégnation, la répétition (à raison de 3 heures par semaine), mais aussi les savoirs, la conceptualisation, l'«apprentissage raisonné », de tradition française : là encore aucune prise de réelle position didactique sur les paris non explicités que la conscientisation ou les degrés de conscience jouent un rôle, quel qu'il soit, dans l'appropriation d'un nouveau système linguistique (Trévise 1996, 2009).

On parle en outre de «maîtrise en langue étrangère », mais peu de choses sont dites sur ce qu'est le langage, et sur ce que représente la violence de la L1 pour un natif, pour qui la conscientisation n'est pas nécessaire, et dont la compétence native peut se résumer par l'utilisation systématique non consciente qu'il fait des oppositions offertes par la langue dite «maternelle », et qui affluent sans cesse automatiquement dans l'apprentissage d'une L2.

Du coup, dans ces textes, au long des années et des réformes, les développements sur les stratégies de l'enseignement et les processus, supposés, de l'apprentissage, égrainent des croyances, avec une masse importante de non-dit, en particulier sur la spécificité de l'appropriation d'une L2 par rapport à la maîtrise de la L1.

La capacité à communiquer en L2 est l'objectif affiché - et le CECRL ${ }^{2}$ la mesure, l'étalonne -, mais on ne dit jamais que, dans la «réalité », hors de la classe, cette communication n'a le plus souvent que faire de la norme syntaxique ou phonologique évaluée en classe et que l'intelligibilité y est le critère majeur. La simulation n'est donc pas véritablement assumée et « l'actionnel» vient encore brouiller les cartes. Jamais n'est véritablement reconnu le statut de langue objet de la L2 en situations de simulation ${ }^{3}$.

Jamais non plus ne ressort clairement ce qui fait progresser des sujets qui ont peu de moyens linguistiques en L2 par rapport à leurs besoins de communication en interaction

\footnotetext{
${ }^{2}$ Cadre Européen Commun de Référence pour les Langues.

${ }^{3} v s$ les DNL (disciplines non linguistiques où l'enseignement de quelques matières se fait en L2), ou les classes d'immersion.
} 
naturelle, i.e. les deux moteurs de tout apprentissage et leurs synergies si complexes :

- l'imitation de l'input en L2 d'une part, et donc la répétition et la mémorisation, quels que soient les moyens employés ;

- la créativité plus ou moins non consciente dans la construction du nouveau système par les apprenants, qui travaille par analogies - et donc transferts interlinguistiques et intralinguistiques, par généralisations. Et ce travail épilinguistique $^{4}$, qui nourrit les stades intermédiaires que sont les interlangues, ne peut - pendant assez longtemps - que partir principalement du filtre préalable de la L1, système qui a façonné la représentation du monde pour tout apprenant, et qui va être inéluctablement transféré, à bon et à mauvais escient, quoi qu'on en pense et quoi qu'on fasse.

Dans ces textes « officiels », l'objectif ultime est ouvertement la communication, et de façon cachée secondaire, presque honteuse, la norme morpho-syntaxique et phonologique, qui doit être également respectée, l'intelligibilité ne sachant suffire. Lexique, phonologie et syntaxe sont abordés au service de la communication, via la culture («entrer dans la langue par la culture »).

Et dans cette « communication », l'argumentation ou l'expression argumentée de points de vue contradictoires représentent toujours l'objectif ultime explicite de la production orale, mais surtout de la production écrite.

Et là, les « connecteurs » - quel que soit les métatermes choisis - ont une place importante.

Ce qui reste néanmoins frappant dans ces textes est que le cours d'anglais semble être le lieu de l'apprentissage de cette argumentation, comme si les sujets n'argumentaient pas en L1, ni à l'oral, ni à l'écrit, comme si, à 16 ou 18 ans, il fallait qu'ils apprennent comment structurer une argumentation, un enchaînement discursif, comme s'il ne leur manquait pas uniquement les moyens - lexique, expressions «figées »- de le faire en L2, comme s'il ne serait pas éminemment producteur que les apprenants transfèrent la façon qu'a le français d'argumenter. Jamais n'est explicité que, pour le stade auquel peuvent prétendre parvenir des apprenants de lycée, une suite discursive argumentative en anglais fonctionne comme en français, en termes cognitifs d'élaborations de causes à conséquences, d'additions d'arguments, d'hypothèses, de concessions.

Or on sait qu'un francophone ne pourra agencer un enchaînement argumentatif véritablement anglo-saxon qu'en parvenant à un stade extrêmement avancé d'apprentissage, voire à l'improbable bilinguisme.

\footnotetext{
${ }^{4}$ définie comme l'activité métalinguistique inconsciente.
} 
Dans les textes, la spécificité de l'anglais n'apparaît que lorsqu'apparaissent des listes «fonctionnelles» utiles pour argumenter et «connecter», ou quand on insiste sur les nécessaires «idiomatismes » - de natures syntaxiques très variées - à mémoriser, et qui représentent l'authenticité même en L2.

Comment est alors présenté cet objectif ultime de la communication, l'argumentation, la confrontation de points de vue ${ }^{5}$, pour le collège et le lycée, ou dans des manuels récents ?

\subsection{Les textes pour le collège}

L'argumentation n'est véritablement abordée qu'en $3^{\text {ème }}:$ dans les «Orientations fonctionnelles», les textes officiels de 2005 font état du «domaine de l'expression discursive », où une des fonctions est de «justifier, commenter, opposer, apprécier, exprimer son accord, son désaccord 》 dans une banque d'expressions intitulée : «lexique et expression figées ${ }^{6} »$ :

Il convient donc, à ce stade, de privilégier l'entraînement à l'expression discursive et à l'argumentation, qu'elle soit orale ou écrite. Pour intensifier les échanges entre les élèves, on choisira des documents de support ou des thèmes de débat qui les conduisent à s'impliquer dans ce qu'ils disent plus qu'ils ne l'avaient fait jusqu'alors, à complexifier leurs énoncés ${ }^{7}$ et à nuancer les points de vue qu'ils expriment. Pour les aider à orienter leur prise de parole dans ces trois directions, on pourra leur fournir des éléments de langage tels que ceux proposés ci-dessous à titre indicatif.

Apparaît donc ici une spécificité réelle de l'apprentissage de la L2 : le manque de moyens linguistiques, et, dans la fonction «Confronter son expérience et sa culture avec celle des autres », sont énumérées - pêle-mêle du point de vue syntaxique - des phrases ou expressions modèles, types, comme :

\section{Although I told the truth, nobody believed me. \\ In spite of all his efforts nothing changed. \\ It's no use panicking, etc.}

Plus loin apparait le titre «Discussion Gambits » qui énumère notamment une liste de fonctions représentées à chaque fois par des phrases ou des expressions «ouvrantes», «idiomatiques», manifestement pour la pratique de l'oral. Nous soulignons les termes qui pourraient réellement être appelés « connecteurs », pour en noter la rareté (but et and mais aussi also et anyway ?) :

\footnotetext{
5 qui s'allie à l'objectif dit « citoyen » de l'ouverture à l'autre...

6 synonyme de «idiomatique »?

7 souligné par nous.
} 
Agreeing with the previous speaker:

I agree with you/him/her entirely/I entirely agree with you.

That's exactly how/what I feel [about it].

That's [just] what I think.

You're/He's/She's right in saying that...

I've never thought offlooked at it that way before, you're right.

That's right.

That's a good point!

That's it!

Exactly!

Disagreeing completely with the previous speaker's view:

I see what you mean but I don't agree at all [with what you/he/she said].

I disagree entirely with...

I don't see it that way at all. I think...

I'm not sure I go along with you on that.

How can you say that?

You can't be serious!/You must be joking!/That's ridiculous!/Rubbish!

I can't believe you support that view/adopt that attitude.

\section{Disagreeing partly and introducing a new argument:}

I see what you mean/he means but...

Yes, but you're forgetting...

We could also say...

Adding something to what the previous speaker has said:

What I'd like to add is...

I'd also like to say...

Anyway, I think...

And what's more/on top of that...

And also, wouldn't you say...

What I find striking/most interesting is...

\section{Raising objections:}

Don't you think it would be better to...?

Yes, but on the other hand...

That's all very well but...

Can I make a point about...?

Other useful phrases in a discussion:

Well..., You know...

There is no point in... [-ing]

You can't make generalisations but...

There are exceptions but...

Well, basically I'm for/against... 
People don't [often/always] realise that...

Nowadays people.../It was different when.../I think it would be better/worse if...

I think [...] is/are responsible [for...]/I think [...] has/have become too...

What I like most is...

I hate/dislike/can't stand...

Suivent l'inévitable mise en garde contre les calques et les moyens recommandés pour l'appropriation, (de l'imitation fréquente aux réflexes, à la spontanéité) :

Apprendre à se servir de ces débuts de phrases, de ces transitions, de ces formules, qui pour la plupart ne sont pas des calques du français, mettra les élèves sur la voie d'une expression plus authentique.

(...) Une phase d'entraînement est indispensable avant que ces amorces d'énoncés ne deviennent des réflexes dans tout acte de communication.

On pourra (...) donner pour tâche aux élèves de réagir, d'argumenter, de justifier et de développer leurs points de vue en utilisant à bon escient ces éléments de langage. La pratique fréquente de tels exercices, de durée limitée, leur permettra petit à petit de parvenir à un certain degré d'autonomie et d'utiliser presque spontanément les formules apprises en amont, dans toute activité orale.

Du côté de la rubrique «grammaire», autre entrée dans les tableaux inclus dans programmes, on découvre une rubrique intitulée : "Opérations ${ }^{8}$ sur des énoncés: l'argumentation». La syntaxe de la phrase simple ou complexe n'est étudiée que du point de vue de la fonction «argumentation», comme si les propositions «adverbiales», et la complexification syntaxique en général, ou la modalisation, n'avaient d'autre fonction que l'argumentation. On y retrouve des « connecteurs », nommés « adverbes » ici, comme still, yet ou however :

Coordination

- Juxtaposition simple ${ }^{9}$ :

- sans adverbe ;

- avec adverbes : still, yet, however...

- Coordination par and, but ou or ; + either... or

- Structures corrélatives comparatives :

- more/-er... and more/-er...

- +the morel-er...the morel-er.

Vient ensuite un programme syntaxique sur la subordination nominale, subdivisée en «relations prédicatives qui prennent la fonction grammaticale d'un nom (complétives en that/Ø, ou des interrogatives indirectes, des infinitives en to, ou des nominales en -ing), d'un adjectif - les relatives - ou d'un adverbe ». Pour cette dernière catégorie, on liste :

\footnotetext{
${ }^{8}$ terme obscur dans une approche non véritablement associée à une théorie linguistique particulière.

${ }^{9}$ rangée ici sous le titre « la coordination »...
} 
Circonstancielles de temps introduites par when, while, as, as soon as, until, before...

Circonstancielles de lieu introduites par where...;

Circonstancielles de cause introduites par because $;+$ since.

Circonstancielles de but introduites par to $;+$ so as to

Circonstancielles de concession introduites par although.

Circonstancielles d'opposition introduites par whereas.

Circonstancielles de condition introduites par if $;+$ unless, provided as long as

Deux sous-rubriques (sémantiques ?) sont ajoutées :

\section{Modalisation}

- Par structure apposée exprimant un commentaire qui porte sur toute la proposition

- Construction en -ing : Frankly speaking,...

- Construction en to : To be frank with you,...

- Complément prépositionnel : Unlike French youngsters,...

- Par structure intégrée exprimant un commentaire qui porte sur toute la proposition

- Verbe au passif (be allowed, be obliged, + be supposed / said /believed)

- + Adjectif à valeur modale (likely, bound sure ...)

Thématisation et focalisation

- Influence de l'ordre des mots et de la place du noyau sur le sens du message.

- Passivation.

- Procédés syntaxiques de mise en relief :

- it is... that/to : It was true that he was fed up with it all ;

- it is... that/who/which : It was in England that I learnt to play darts.

On ne sait plus ce que veut dire «argumenter » dans l'esprit des rédacteurs par rapport à parler ou écrire, et la syntaxe est manifestement mise au service des seules visées argumentatives.

2.2. Au lycée ${ }^{10}$ : de la juxtaposition d'énoncés simples à l'argumentation

Les mots clés dans le texte de 2004 sont: enchaînements, cohérence, mots de liaison, complexification, connexions, connecteurs, balises, articulations logiques, indices.

On ne trouve plus d'entrée fonctionnelle aux structures censées être utiles pour

\footnotetext{
${ }^{10}$ Rappel : l'ensemble de la grammaire et du lexique est censé être vu à la fin du collège et donc au lycée il n'y a que des révisions.
} 
l'argumentation. Par contre on a longuement accès aux représentations du Ministère et du CNDP sur l'apprentissage/enseignement des langues étrangères, notamment sur l'objectif ultime qu'est l'argumentation.

Dans la rubrique LV3 des Programmes apparaît l'objectif ultime, celui d'un anglais « idiomatique », tandis que, dans le préambule commun précédant les programmes de seconde pour toutes les langues vivantes et concernant l'expression orale, il est question pour l'élève de :

savoir, tant sur le plan de la syntaxe que sur celui de la prosodie, enchaîner des énoncés entre eux d'un point de vue chronologique (antériorité, simultanéité, postériorité), d'un point de vue logique (enchaînement cohérent, inattendu, illogique, etc.) en utilisant les mots de liaison les plus courants entre phrases (...). En fin de seconde, l'élève (...) peut enchaîner des énoncés pour donner cohérence à sa pensée et ses opinions, et susciter un éventuel débat avec son interlocuteur. ${ }^{11}$

Les termes de «proposition», «phrase » ou «énoncé » sont synonymes manifestement. Pour l'expression écrite il est stipulé :

On aura toujours présent à l'idée que la cohérence discursive reste le principe organisateur du texte. (...); l'élève apprend en LV1 à complexifier de plus en plus ses énoncés et à les enchaîner en fonction de ses besoins d'expression personnels.

Pour la compréhension de l'oral, dans la définition des objectifs, on parle de stratégies d'écoute active qui consistent pour l'élève à :

repérer les indices de cohérence (repères spatio-temporels, articulations logiques) afin d'affiner la compréhension du message.

Ce repérage ne peut qu'être issu d'une activité métalinguistique d'observation et de découpage de l'input à des fins tant d'imitation que de conceptualisation. Pour la compréhension de l'écrit, de même, l'élève devra également :

identifier les valeurs exprimées par les formes grammaticales (comparaison, modalité, passivation, etc.) pour faire des suppositions et vérifier leur justesse en cours de

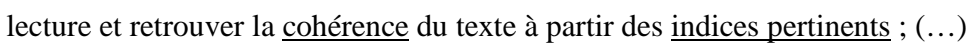

Un véritable entraînement, ici aussi, mettra en place des stratégies de lecture adaptées au type de document rencontré : repérage de l'agencement argumentatif dans les textes discursifs, de la trame narrative dans les récits, des points d'ancrage de la communication dans les publicités et les brochures.

\footnotetext{
${ }^{11}$ Nous prenons le parti de souligner tout ce qui relève de la métalangue de la « discursivité », de la « cohérence discursive », plus généralement des « connexions».
} 
En terminale, pour la lecture en continu, les consignes sont quasi identiques.

Écouter et lire vont être la source de ces activités d'imprégnation (le «bain de langue »), mais aussi de conscientisation, de construction de repères métalinguistiques. Imitation et créativité en seront accrues, espère-t-on. On peut d'ailleurs lire plus loin, quand il s'agit aborder «l'expression discursive et l'argumentation » :

Pour ce faire, il s'agit d'entraîner à l'analyse du discours oral (...) il s'agit de sensibiliser les élèves à la créativité de la langue et de les inciter à apprendre des mots, des expressions ou des énoncés préformés pour créer une expression personnelle et originale.

Par cœur et créativité doivent s'allier, mais on insiste beaucoup sur la mémorisation d'expressions de L2.

Pour ce qui est de la production écrite, testée au baccalauréat, et qui garde sa place en haut de la hiérarchie des productions, on peut lire :

En classe de seconde, les activités proposées devront amener les élèves à (...) produire des énoncés d'une complexité croissante.

(...)

Le pastiche est un exercice à envisager, "écrire à la manière de..." permet d'établir un lien entre compréhension et production. L'enseignant pourra en cours d'année commencer à travailler l'argumentation.

On attendra des élèves qu'ils soient capables de construire des paragraphes et au final de rédiger des productions de 150 mots environ.

Pour cette compétence, qu'il s'agisse de la rédaction d'énoncés simples ou d'une production écrite plus complexe, l'élève est amené à mettre en œuvre certaines stratégies productives, parmi lesquelles :

(...)

- sélectionner les mots de liaison afin de structurer les paragraphes et de créer la cohérence de l'ensemble de la production ;

- complexifier et enrichir le discours pour exprimer une pensée argumentée, étoffée et nuancée ;

- contrôler la production en utilisant ses connaissances pour identifier et corriger ses erreurs.

Dans le cadre du travail écrit, il conviendra de faire ressortir les spécificités de l'oral et de l'écrit : alors qu'à l'oral on privilégie la juxtaposition d'énoncés simples ${ }^{12}$, la langue écrite permet la production d'énoncés complexes faisant appel aux processus de subordination syntaxique.

\footnotetext{
${ }^{12}$ La langue orale est ainsi stigmatisée, à plusieurs reprises dans ce long texte.
} 
- rédiger un texte court à caractère narratif, ou développant une argumentation (phrases complexes, paragraphes, articulations rhétoriques) ;

La L2 n'a donc aucune spécificité ici. Mais on incite les élèves à l'autocorrection - à l'écrit uniquement - en ces termes étranges :

Le discours écrit étant plus précis et plus structuré que le discours oral, l'enseignant doit former les élèves à l'autocorrection.

Selon l'objectif poursuivi, les erreurs ciblées pourront être de toutes natures : linguistiques (morphologie, choix des marqueurs...), logiques (articulations, cohésion textuelle...), rhétoriques (distribution des thèmes, organisation du discours, construction des paragraphes...), voire narratologiques, dans les textes de fiction.

Pour la classe de première, on lit aussi à propos de l'expression écrite :

elle suppose un temps de réflexion plus long et une organisation plus élaborée des énoncés. Ceux-ci se complexifient en incorporant les outils propres à l'organisation chronologique et argumentative (par exemple, "avant que", "après que", "bien que"13) qui nécessitent selon les langues des réaménagements temporels, modaux entre autres. Ils s'organisent en paragraphes, puis en brefs textes autour d'un thème de réflexion. Les enchaînements sont plus cohérents, le vocabulaire le plus précis possible.

Subordination, coordination, mots de liaison : tout sert à l'«enchaînement», l'« organisation », la «cohérence ». Tout vaut mieux que la parataxe asyndétique, code restreint s'il en est, tout juste bonne pour l'oral. Rien n'est dit ni sur une spécificité de la L2, ni sur le fonctionnement de tout ceci en L1 (qui est tabou ?).

Toujours pour cette classe on lit plus loin :

L'organisation morpho-syntaxique de la phrase est mise en relation avec l'organisation du texte, les mécanismes de sa cohérence et de son intelligibilité, et audelà, avec le type de discours, la situation d'énonciation, sa dimension pragmatique.

Et enfin la note finale :

L'objectif est de construire une pratique communicative de la langue, induisant chez l'élève des comportements discursifs destinés à décrire, convaincre, raconter, expliquer, argumenter, débattre, etc., comportements dont l'évaluation doit mesurer le degré de maîtrise.

\footnotetext{
${ }^{13}$ Conjoncteurs cités en français...
} 
Sur la « discursivité », en terminale, à propos de la production orale et écrite, on est quelque peu étonné de lire encore :

Sur le plan syntaxique, on approfondira le travail effectué les deux années précédentes, en privilégiant plutôt, à l'oral, la coordination, les adverbes (yet, $\underline{\text { though } . . .)^{14} \text {, les relatives déterminatives, etc., et à l'écrit, la subordination, les }}$ relatives descriptives ${ }^{15}$, etc.

Tout est résumé à propos des « débats » mis en place en classe de terminale, pour des sujets qui font par ailleurs - il est nécessaire de le rappeler - des dissertations de philosophie ou de français :

À cet égard, l'apprentissage de la nuance impliquera le recours fréquent à la modalisation, à la concession, aux quantifieurs, aux pivots logiques. Moduler sa propre position et reformuler celle de l'autre seront ainsi deux des principaux objectifs visés.

Quid ici et de la spécificité de l'anglais et des processus d'apprentissage ? D'un appui sur des compétences langagières censées être acquises à 18 ans ?

Sont-ce des conseils de développement cognitif pour des francophones qui ne sauraient pas argumenter avant de rentrer dans la classe d'anglais?

On aura remarqué que le mot « connecteur » n'apparaît jamais ${ }^{16}$.

Dans le texte à propos des épreuves du baccalauréat, LV1, on trouve le terme de « connecteur »:

Est évaluée essentiellement l'aptitude du candidat à rédiger, dans une langue correcte et directement compréhensible, un ou des textes cohérents clairement articulés à l'aide de connecteurs logiques et chronologiques simples et courants (niveau B1 du CECRL)

Cependant, les concepteurs de ces textes connaissent les écueils fréquents dans les productions des élèves («erreurs » dues à un enseignement behavioriste trop intensif de formules, plutôt que conceptualisant et contrastif ?), et ils avertissent :

\footnotetext{
${ }^{14}$ que certains nommeraient « connecteurs »... 15 ?

16 Notons que pour le CECRL, l'expression «connecteur simple » renvoie manifestement aux conjoncteurs de coordination et de subordination, qui côtoient des connecteurs (moins simples?) logiques ou chronologiques.
} 
En même temps, un débat ne doit pas devenir une simple joute oratoire, une machine rhétorique vide.

$(\ldots)$

le travail de production écrite vise à la mise en place d'énoncés cohérents et complexes sans pour autant donner lieu à des caricatures de discours où l'abondance de mots de liaison stéréotypés tente de masquer l'indigence de la langue. L'utilisation adéquate de la subordination syntaxique, de la coordination et la précision lexicale devront permettre à l'élève de parvenir à une expression riche, nuancée, et surtout naturelle $e^{17}$.

On observe effectivement fréquemment des copies où le squelette des expressions argumentatives mémorisées encadre des phrases vides de contenu.

\section{3. Illustrations de mises en œuvre : quelques exemples tirés de manuels} récents

Dans Good news, classe de $3^{\text {ème }}(2009,161)$, dans le Précis Grammatical, après une rubrique concernant la subordination, apparaît une dernière une rubrique, où les exemples sont traduits en français, qui s'intitule : «les mots de liaison». Adverbes, conjonctions entre propositions, entre noms, sont mêlés. On est surpris par la confusion de l'ensemble :

\section{Les mots de liaison}

- And associe ou ajoute

John and Mary have been married for five years.

John et Mary sont mariés depuis cinq ans.

- But signale un contraste

She's rich but she's unhappy.

Elle est riche mais elle est malheureuse.

- Firstly (second[ly], third[ly], etc.), then, finally indiquent les étapes d'une suite chronologique :

First, l'll talk about last year's results, then l'll tell you...

Premièrement, je vous parlerai des résultats de l'année dernière, puis je vous dirai...

Finally, I'd like to ask you a question. Enfin, j'aimerais vous poser une question.

- So marque un lien de cause à effet:

He was ill so he couldn't play football ${ }^{18}$. Il était malade, alors il ne pouvait pas jouer au football.

- Because apporte une explication

She can't come because she's working tonight. Elle ne peut pas venir parce qu'elle travaille ce soir.

17 synonyme d' « idiomatique »?

18 L'absence de ponctuation dans l'exemple avec so laisse perplexe : la virgule apparait bien dans la traduction française. 
Dans le manuel de $2^{\text {nde }}$, On Target, qui s'annonce tout à la fois comme une «approche communicative et actionnelle et une «approche énonciative et communicative dans l'explication des faits de langue », on voit un encadré «language corner», avec une rubrique : «les conjonctions utiles pour l'argumentation: even, if, unless ». Deux exemples sont donnés, suivis de la question: "Quelle notion(s) chacune des deux conjonctions introduit-elle dans ces exemples pour mettre deux éléments en parallèle ?». Dans la même disposition graphique on avait eu :

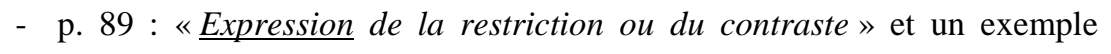
faisant figurer, sans en nommer la catégorie, la préposition despite.

- et p. 65 «Cause et conséquence ».

a. ... my arms were tawny and strong from working long days on the shrimp boat in the Blazing South Carolina heat.

b. Because I was a Wingo, I worked as soon as I could walk.

c. My father beat me for breaking the law.

Ces exemples invitent à une réflexion plus intéressante : «Indiquez pour chacune de ces phrases ci-dessus quelle partie exprime la cause et quelle partie exprime la conséquence? Indiquez le mot qui introduit la notion de cause. »

Renvoi est fait au précis grammatical en fin d'ouvrage où les «mots de liaison» n'apparaissent plus que comme des catégories grammaticales: «conjonctions de coordination ou adverbes » comme yet, however, so, therefore, etc., et conjonctions de coordination («les plus courantes » : and, but, either (or) neither (nor), now, whether (or)). Les relatives sont traitées comme des propositions subordonnées, à côté des subordonnées nominales ou adverbiales. Puis vient l'explication :

\footnotetext{
D'autres outils de liaison sont également utilisés : Ø ou l'opérateur ${ }^{19}$ to.

$\emptyset$ marque en général le fait qu'un mot de liaison a été omis ou que la liaison se fait sans mot-outil. (p. 190)
}

On peut lire deux pages plus loin la rubrique bilingue, intitulée : «Les propositions conjonctives et les conjonctions de subordination », dont il est dit qu'elles «apportent des informations de diverses natures »:

\author{
Le temps (when, after as since, till, until) \\ Le but (in order to so as to, so that) L'opérateur TO peut suffire à introduire le but. \\ La condition : if, provided, unless, as long as, = (si, à condition que), unless (a moins \\ que), as long as (à la condition que) \\ Le contraste : wheras (tandis que) \\ La cause : as : because, since
}

\footnotetext{
${ }^{19}$ Métaterme brusquement issu d'une pseudo approche « énonciative »...
} 
La crainte, le risque : for fear, lest, (de peur que, au cas où)

La concession, l'opposition, la restriction : although (bien que), though (bien que),

even though (même si), even if (même si)

En fin de manuel (p. 210), dans un précis intitulé «méthodologie », apparaît enfin une rubrique qui fournit la banque de mots ou d'expressions. Il ne s'agit pas de «connecteurs », mais d' «expressions et tournures idiomatiques (= typiques de la langue anglaise) 》 pour «organiser et lier son discours ». Il est «fortement conseillé d'y insérer des mots de liaison, notamment à l'écrit, mais pas seulement. ».

Suivent des listes bilingues de conjonctions, adverbes, expressions organisées en fonctions (avec le français en premier) et non en catégories qui commanderaient leur utilisation :

\author{
Pour commencer par une généralitée \\ Pour clore un débat ou une argumentation \\ Pour exprimer son point de vue \\ Pour exprimer une conséquence \\ Pour compléter ou préciser son argumentation \\ Pour mettre deux éléments en parallèle \\ Pour exprimer une contradiction, une opposition
}

Dans Meeting Point, classe de $2^{\text {nde }}$, la seule référence unilingue à l'argumentation est la suivante :
Donnez votre opinion
As far as I am concerned, In my opinion, to my mind, it reminds me of, it looks like...
(p. 253).

Enfin, dans Step In, $3^{\text {ème }}$, la rubrique «Méthodologie» (p. 129), est la seule à faire référence à l'argumentation :

\footnotetext{
Organise tes phrases en paragraphes, ce qui rendra ta production plus aérée et agréable à lire. Utilise des mots de liaison pour organiser tes idées (first, then after that, finally, so, that's why, moreover (de plus) in fact) ; ils te permettront de structurer tes idées et d'éviter une simple succession de phrases sans lien apparent. Efforce-toi d'avoir une expression écrite riche et variée.

Réfléchis à l'enchaînement des idées avant de passer à la rédaction. Prévois de créer un paragraphe par idée ou par argument.
}

\footnotetext{
${ }^{20}$ Un exemple : Il est indéniable que... There is no denying that...,
} 
Ce qui frappe le plus, pour ce qui concerne la L2, est la non prise en compte des capacités à argumenter et à utiliser les «connecteurs » en L1. Mais il est vrai que la L1 - pourtant omniprésente pour les apprenants - n'a guère droit de cité dans cet enseignement et l'invocation quasi-magique de l'idiomaticité ne saurait résoudre le problème de la nécessaire contrastivité et de sa conscientisation.

\section{Quelques remarques conclusives et programmatiques}

Même si les linguistes eux-mêmes ont du mal à définir ce qu'est un «connecteur », cette utilisation de l'expression «mot de liaison », ces recours sporadiques et non systématiques aux catégories grammaticales, font encore une fois questionner la métalangue d'enseignement en L1 comme en L2.

Les termes métalinguistiques, loin de s'organiser s'ajoutent et se superposent conjonctions, mots de liaison, connecteurs - sans clarifier les fonctionnements respectifs dans les deux langues et l'enseignement de la L2 ne s'appuie pas sur la métalangue diffusée depuis le primaire - certainement à revisiter - pour celui de la langue «maternelle». Les enseignants, comme les élèves, se retrouvent devant un maquis de termes qui suscitent la confusion, voire la suspicion. L'extension sémantique de ces termes étant floue, on privilégie, en L1 comme en L2, l'apprentissage de listes hétérogènes plutôt que la réflexion sur les relations qu'instaurent lesdits « connecteurs ». On retrouve là à la fois une tradition analytique française et une conception manifestement behavioriste.

En effet, que l'on puisse intégrer «That's why...», «Frankly speaking...» ou «Nous en déduisons que...» dans une pseudo-catégorie «mots de liaison» montre bien que la volonté d'adopter un point de vue textuel et argumentatif, pour l'étude tant de la L1 que de la L2, écarte une nécessaire conscientisation de la syntaxe (y compris dans la formation des enseignants...). Apparait alors une approche sémantique floue qui brouille les catégories grammaticales et syntaxiques. Des expressions comme «mots de liaison», «mots permettant l'agencement des parties entre elles », ou donc «connecteurs » englobent bien des syntagmes prépositionnels ou des propositions intra- et extra-prédicatifs, mais aussi des propositions entières, antéposées le plus souvent, et apprises sans référence à une quelconque manipulation paradigmatique. Par exemple en L1, on fait relever aux élèves l'expression de la cause, de la conséquence ou de la concession, sans leur donner les moyens de délimiter les segments mis en relation, ni de distinguer ce qui relève de la progression du texte ou de l'organisation de la phrase. La cohérence discursive et les continuités ou ruptures thématiques ne sont guère abordées. Prise en charge énonciative et argumentation entrent pêle-mêle dans une approche dite textuelle et voulue pragmatique.

Là est le paradoxe : bien que la «maîtrise de la langue » soit devenue l'évidente finalité explicite des apprentissages linguistiques et langagiers en L1 comme en L2, les emplois 
effectifs des connecteurs par les élèves, en L1 à l'oral comme à l'écrit, sont ignorés. Ce qui frappe aussi c'est une prise en compte de l'oral qui se contente de normativité - parcellaire, voire erronée - et n'inclut nullement une macrosyntaxe alors que c'est dans ce cadre que les unités discursives d'articulent aux connecteurs à l'oral comme à l'écrit mais selon des modalités différentes.

On ne rencontre pas d'exercices qui s'appuient sur la connaissance qu'ont les élèves du fonctionnement des connecteurs en L1 pour clarifier des emplois dans une approche contrastive dans chacune des langues et d'une langue à l'autre: on pourrait par exemple faire confronter dans différents contextes et à différents niveaux parce que et puisque et les comparer à because et since, toutefois et cependant vs however, comme à valeur causale vs as.

Les langues ne sont plus abordées en tant que systèmes et les approches contrastives réflexives en L2 sont occultées au profit du culte de l'idiomaticité, comme si celle-ci excluait toute systématisation et comparaisons entre les deux systèmes.

Or la spécificité de l'Ecole, par rapport au milieu dit «naturel» ou «non guidé », est justement l'accès aux savoirs en L1 et en L2, et non simplement aux savoir-faire, et on ne saurait donc faire l'économie d'une conscientisation de la (morpho-)syntaxe des deux langues ni de la contrastivité, sachant que le système préalable de la L1 reste prégnant et omniprésent dans ses aspects facilitateurs mais aussi générateurs de calques.

Cette approche pseudo-pragmatique exclut apparemment la syntaxe - rébarbative ? - de toute fonctionnalité textuelle et communicative. On prétend enseigner une complexification de la pensée et de l'expression sans enseigner la complexification syntaxique dans les systèmes.

Comment dès lors s'étonner que la phraséalogie plaquée en L1 et en L2 vienne remplacer une articulation des contenus qui fasse réellement sens ? Et ceci est d'autant plus choquant en L2 au lycée que l'on a affaire à des apprenants qui possèdent les compétences cognitives et métacognitives de l'argumentation, mais à qui manque justement la maîtrise de la L2 en tant que système et non simplement en tant qu' « outil de communication».

$\mathrm{Si}$ savoir et réflexion métalinguistiques il doit y avoir - et c'est le rôle de l'Ecole comment ne pas s'interroger encore une fois - et c'est le vrai défi pour les didacticiens (Elalouf \& Trévise 2009) - sur une utilisation de métatermes communs, simples mais justes, qui soient clairement définis pour les apprenants et qui fassent sens pour eux, qui ne nient pas les catégories grammaticales et syntaxiques, qui tentent de clarifier les liens entre agencement syntaxique et (re)construction du sens ? 


\section{Références bibliographiques}

Arrivé, M., Gadet, F., \& Galmiche, M. (1984). La grammaire d'aujourd'hui. Paris, Flammarion.

Combettes, B. (2009). Quelle(s) description(s) grammaticales(s) pour l'enseignement? . Repères $\mathrm{n}^{\circ} 39,41-56$.

Elalouf M.-L. (éd.) (2009). Le groupe nominal et la construction de la référence. Descriptions pour l'enseignement du français et d'autres langues. Diptyque 16, Presses Universitaires de Namur, 16-28.

Garcia-Debanc, Cl. (1998). Transpositions didactiques chaînes de reformulation des savoirs : le cas des connecteurs. La transposition didactique en français, Pratiques $\mathrm{n}^{\circ} 97-$ 98, 133-152.

Maingueneau, D. et Pellet, E. (2005). Les notions grammaticales au collège et au lycée, guide Belin de l'enseignement. Paris, Belin.

Moeshler, J. \& Auchlin, A., (2009). Introduction à la linguistique contemporaine. $3^{\mathrm{e}}$ éd. Paris, Armand Colin.

Riegel, M., Pellat, J.-C., Rioul, R. (1994). Grammaire méthodique du français. Paris, PUF.

Masseron, C. (2004). Les paradoxes de CAR, entre énoncés et discours - ou les difficultés d'un traitement didactique des connecteurs. In Anis J. et Boré C. (éd.),Linx n51, Théories de l'écriture et pratiques scolaires, Paris X-Nanterre, 107-128.

Schneuwly, B. (1989). Les organisateurs textuels dans quatre types de textes écrits (élèves 10, 12, 14 ans). Langue française, 81, 40-58.

Touratier, C. (2000). Que faut-il entendre par connecteur? http://sites.univprovence.fr/wclaix/connecteurs/connecteurs.htm

Trévise, A. (1996). Réflexion, réflexivité et acquisition des langues. In Trévise A. (éd.), A.I.L.E. $\mathrm{n}^{\circ} 8$ : Activités et représentations métalinguistiques dans les acquisitions des langues, 5-39.

Trévise, A. (2009). Les textes officiels français pour les langues vivantes et l'enseignement/apprentissage de la grammaire. L'exemple de la détermination nominale en anglais. In Elalouf M.-L. (éd.), Diptyque 16, Presses Universitaires de Namur, 103-124.

Textes officiels pour l'anglais

Enseigner au collège, anglais LV1 et LV2. Programmes et accompagnements, réédition 2005.

Programmes et Accompagnements qui réunit les programmes d'enseignement de l'anglais en classe de seconde, de première et terminale de la voie générale et technologique et les accompagnements des programmes des classes de seconde, de première et terminale de la voie générale et technologique (BO hors série $\mathrm{n}^{\circ} 5$ du 9 septembre 2004 volume 15 et $\mathrm{JO}$ du 17 juillet 2004).

Manuel de français 
L'atelier du langage, Hatier, 2009.

Manuels d'anglais

Good news, $3^{\text {ème }}$, Belin, 2009.

On target, $2^{\text {nde }}$, Belin, 2009.

Meeting Point, $2^{\text {nde }}$, Hatier, 2009.

Step in, $3^{\text {ème }}$, Hatier, 2009

Marie-Laure Elalouf

Marie-laure.elalouf@iufm.u-cergy.fr

22 rue Armand Carrel

92160 - Antony

Anne Trévise

anne.trevise@wanadoo.fr

18 rue du Colonel Oudot

75012 - Paris 